\title{
Razonamiento funcional y causal sobre el desequilibrio ecosistémico en estudiantes universitarios
}

\section{Functional and causal reasoning about the ecosystem imbalance in university students}

\author{
Joan Gil-Contreras \\ Claudia Navarro-Roldan \\ Iván Pinzón-Prieto \\ Universidad Pedagógica y Tecnológica de Colombia, Tunja, Colombia
}

\begin{abstract}
Resumen
Objetivo: describir cómo los estudiantes universitarios razonan funcional y causalmente sobre un ecosistema, usando el modelo estructura, comportamiento y función. Método: se acoge un diseño transversal descriptivo, con un modelo mixto de triangulación concurrente; simultáneamente se recolectaron y analizaron datos cualitativos y cuantitativos para compararlos e integrarlos. A partir de muestreo intencional con estudiantes universitarios $(n=60)$ se conformaron cuatro grupos: estudiantes de Biología y de Licenciatura en Ciencias Naturales y Educación ambiental. Los participantes se dividieron en cuatro grupos equitativos, de acuerdo a la carrera y a la condición de haber cursado o no durante el 2018 dos asignaturas obligatorias del currículo relacionadas con la ecología. Ellos respondieron una entrevista sobre ecosistemas y resolvieron una situación problema planteada en un bioterio afectado por actividades antropogénicas y residuos contaminantes. Resultados: se encontraron diferencias atribuibles al nivel de experticia entre los estudiantes de la carrera biología que cursaron ecología, quienes comprendieron el ecosistema desde la interdependencia dinámica de la estructura y las funciones, frente a otros grupos, que enfatizaron en los componentes estructurales con mayor saliencia perceptual. Conclusiones y discusiones: aunque la mayoría de los estudiantes comprenden las relaciones causales y funcionales del ecosistema, existen algunos sesgos en la construcción conceptual, los cuales pueden ser explicados desde el reconocimiento incorrecto de la causa y el establecimiento de relaciones causales.
\end{abstract}

Palabras clave: ecosistemas, estudiantes universitarios, razonamiento, sesgos explicativos, sistemas complejos.

\begin{abstract}
Objective: this paper describes how university students argue functionally and causally about an ecosystem, using the Structure, Behavior and Function models. Method: a descriptive transversal design has been used with a mixed model of concurrent triangulation. Simultaneously, qualitative and quantitative data have been gathered and analyzed in order to compare and integrate them. From intentional sampling with college students $(n=60)$, four groups have been formed: biology students, and natural sciences and environmental education students. They have been divided into four equal groups according to their studies and to the condition of having completed or not during year 2018 the two mandatory subjects related to ecology. They answered interview questions about ecosystems and resolved a situation problem raised in a bioterium affected by anthropogenic activities and contaminating residues. Results: we found differences attributable to the level of expertise between biology students that studied ecology, who understood the ecosystem from the dynamic interdependence of structure and functions, and other groups, who emphasized structural components with greater perceptual salience. Conclusions and discussion: although most students understand the causal and functional relationships of the ecosystem, there are some biases in the conceptual construction, which can be explained from the incorrect recognition of the cause and the establishment of causal relationships.
\end{abstract}

Key words: ecosystems, university students, reasoning, explanatory biases, complex systems.
Open Access:

\author{
ISSN: 0124-2121 \\ E-ISSN: $2665-2420$ \\ ARTÍCULO RESULTADO DE
INVESTIGACIÓN \\ Copyright $\odot$ \\ By Educación y Humanismo
}

Editor:

Dhayana Fernández Matos

Universidad Simón Bolívar

Correspondencia:

claudia.navarro@uptc.

edu.co

Recibido: 13-04-2020

Aceptado: 20-05-2020

En línea desde: 17-07-2020

DOI:

10.17081/eduhum.22.39.4 


\section{Introducción}

Un sistema complejo es un sistema compuesto por múltiples elementos o agentes que interactúan entre sí (e.g., en el nivel micro de un ecosistema los animales y plantas) y con su entorno (e.g., en el nivel macro de un ecosistema el flujo de energía o la condensación del agua). Las interacciones entre los agentes del sistema se auto-organizan y transforman el comportamiento global o emergente observable del sistema. Sin embargo, este comportamiento emergente no es intencional y predecible desde el comportamiento de los agentes en el nivel micro; por el contrario, es azaroso, no predecible, evoluciona, no tiene fin, pequeños cambios pueden generar grandes consecuencias, o viceversa, entre otras características constitutivas (García, 2011, 2006; Hmelo-Silver \& Green, 2004; Jacobson, 2001). En este sentido, comprender un sistema complejo es difícil porque requiere pensamiento abstracto $y$, con frecuencia, desafía las creencias del funcionamiento determinista o intuitivo de las relaciones entre los niveles micro y macro de diferentes fenómenos en las ciencias naturales (Ferrari \& Chi, 1998; Hmelo-Silver\& Green, 2004).

Los estudiantes universitarios sesgan su proceso de razonamiento científico cuando incorporan en su representación mental o, en su modelo conceptual o mental, algunos atributos y/o componentes, o relaciones entre ellos, que no dan cuenta de la constitución o funcionamiento del sistema complejo que pretenden explicar (Chi, 2005; Goel, Goméz, Grúe, et al. 1996; Perkins\&Grotzer, 2000; Wilensky\&Resnick, 1999). En las ciencias naturales, por ejemplo, cuando los estudiantes no discriminan y articulan la totalidad de las relaciones causales e interdependientes que existen entre los niveles y agentes de un sistema complejo en funcionamiento, entonces, ellos sesgan su razonamiento disciplinar (D’Avanzo, 2003; Jeffries, Stanisstreet\& Boyes, 2001; Kevin \&Munson, 2008; Shepardson, 2005; Tekkaya, 2002).

Por ejemplo, Wilensky y Resnick (1999) encontraron que, al explicar los sistemas ecológicos, los estudiantesconstruyen sesgos porque no interrelacionan los genes, los individuos, las poblaciones y las especies, al mismo tiempo queestablecen relaciones causales directas entre el comportamiento individual de un organismo (a nivel micro) y el comportamiento emergente enel medio ambiente en su conjunto (a nivel macro). Por su parte, Chung, Hogan, Brazis, et al. (2000) argumentan que, los estudiantes universitarios construyen sesgos al razonar sobre los procesos de flujo de energía y cadenas alimenticias en un ecosistema porque ellos representan las redes tróficas en términos de cadenas alimenticias individuales con efectos directos y predecibles, en lugar de representarlas como cadenas interconectadas y comportamiento azaroso.

Estos sesgos en el razonamiento causal de los sistemas complejos pueden explicarse porque los estudiantes comprenden los sistemas desde modelos mentales intuitivos (e.g., a partir del razonamiento inductivo, modelo determinista unicausal, en la percepción, en la experiencia, en autoexplicaciones, en estereotipos, en evidencia no corroborada); 
dejando de lado modelos científicos que permitirían elaborar explicaciones plausibles a nivel disciplinar (e.g., basados en la lógica formal deductiva, modelos multicausales, en la evaluación de hipótesis, en experimentación metódica, en la evidencia contrastada) (Chi, 2005; Chi, Slotta\& De Leeuw, 1994). Para evitar el sesgo debería existir correspondencia entre el modelo mental usado para explicar el funcionamiento causal en un sistema complejo y las características dinámicas que constituyen el sistema.

Estudiantes universitarios de Biología sesgan la construcción conceptual de los sistemas complejos en un hábitat (i.e., la explicación sobre cómo las características del lugar impactan en la sobrevivencia de organismos), cuando ellos omiten las relaciones interdependientes entre los componentes bióticos y abióticos (Shepardson, 2005) 0, cuando privilegian las características estructurales y funcionales de un hábitat como refugio de una solo especie, omitiendo las interrelaciones a nivel de comunidades o poblaciones que comparten un hábitat (Sánchez \& Pontes, 2010). Estudios previos sobre la comprensión del sistema de respiración humano (Hmelo, Holton, \& Kolodner, 2000), los ecosistemas (Hmelo \& Green, 2004) o el ciclo de vida del moho limo (Wilensky \& Resnick, 1999) revelan que, los estudiantes universitarios usan modelos mentales intuitivos y perceptuales para representar las relaciones multinivel y emergentes con las que se comportan los sistemas complejos; $y$, en consecuencia, ellos explican desde modelos mentales directos, unicausales y predecibles, que no permiten dar cuenta de su funcionamiento dinámico y multinivel.

En esta línea, las preguntas de investigación que orientaron este estudio fueron: ¿cómo los estudiantes universitarios construyen epistémicamente el funcionamiento de un ecosistema? y ¿cuáles son los sesgos causales en sus construcciones conceptuales? Para dar respuesta, se adoptó el modelo de análisis Estructura, Comportamiento y Función (ECF) usado para comprender la construcción de conceptos sobre sistemas complejos en áreas de la física (Goel, et al. 1996; Weld, 1983) y la biología (Hmelo \& Green, 2004; Hmelo, Holton \& Kolodner, 2000). El modelo ECF incluye tres componentes: a) estructura (E) o los elementos o agentes del sistema (e.g., plantas, animales, factores bióticos, etc.); b) comportamiento (C) referido a cómo los elementos o agentes de un sistema logran su propósito, cómo interactúan o cuáles mecanismos informan de un producto, reacción o un resultado (e.g., las plantas transforman la energía solar en energía química para producir su alimento); y c) función (F) o por qué existe un elemento dentro de un sistema, cuál su propósito dentro del sistema (e.g. las mariposas son polinizadores, plantas filtran el agua, etc.).

En la ecología, los ecosistemas ontológicamente se constituyen como un sistema complejo porque guardan un comportamiento dinámico, multinivel y emergente (Hogan, 2000). El ecosistema se transforma y auto-organiza de forma permanente para lograr un equilibrio constante, a partir de interacciones regladas y descentralizadas de redes de múltiples agentes estructurales que lo componen en el nivel micro (e.g., microrganismo, 
aves, mariposas, gusanos). Por su parte, el comportamiento de los organismos en el nivel micro en el ecosistema, guarda interdependencia funcional con el nivel macro del ambiente global del sistema y otros sistemas o especies, es decir, aquellos comportamientos observables del ecosistema (e.g., flujo de energía, condensación del agua) (Ferrari \& Chi, 1998; Perkins \& Grotzer, 2000; Wilensky \& Resnick, 1999).

No identificar las características (o atributos) ontológicas de los sistemas complejos se asocia a la construcción de sesgos en el razonamiento (Chi, et al. 2012). Por ejemplo, Jacobson (2001) entrevistó a estudiantes y profesores universitarios sobre los sistemas complejos (e.g., hormiguero) encontrando que, mientras los estudiantes basan su razonamiento en un sistema causal simple, de control central, con resultados predecibles; los profesores usaron modelos que, ontológicamente, corresponden a la complejidad de sistema: descentralización, la multicausalidad, procesos estocásticos y de equilibrio permanente. Consistentemente, múltiples estudios (Astudillo \& Gene, 1984; Bahar, 2003; Eyster \& Tashiro, 1997; Hogan \& Fisherkeller, 1996; Hungerford \& Volk, 1990; Mahmud \& Gutiérrez, 2010) revelan que, los estudiantes construyen sesgos al explicar los sistemas complejos porque: a) privilegian la organización de sistemas en niveles jerárquicos con interacción centralizada y local; b) se centran en elementos estructurales del sistema con mayor saliencia perceptible; y c) sobrecargan la memoria de trabajo al no segmentar las múltiples interacciones simultáneas del sistema.

Por ello, el objeto de este estudio es identificar las formas en que los estudiantes, que se forman para ser futuros maestros, razonan funcional y causalmente sobre los problemas ambientales que involucran ecosistemas. Se espera aportar insumos en la comprensión sobre cómo se construye el conocimiento biológico en las aulas universitarias y posibilitar cambios en las prácticas de aula (Kevin \& Munson, 2008; Munson, 1994) que posibiliten el uso de la complejidad para acercarse a la comprensión de los fenómenos naturales.

\section{Método}

\section{Diseño}

Se usó un diseño transversal de tipo descriptivo, aplicando un modelo mixto de triangulación concurrente. Siguiendo a Hernández-Sampieri, Fernández y Baptista (2010), los modelos mixtos permiten recolectar y analizar datos cualitativos y cuantitativos de forma simultánea para compararlos e integrarlos; en esta medida posibilitan la integración de los datos para discutirlos de manera conjunta y establecer inferencias sobre el fenómeno estudiado. Adicionalmente, el modelo de triangulación concurrente permite la validación cruzada entre los datos cuantitativos y cualitativos. 


\section{Participantes}

La muestra estuvo conformada por 60 estudiantes universitarios seleccionados a través de un muestro intencional por conveniencia, entre los 18 y 28 años de edad $(M=21$; $D E=1,94)$, 18 hombres y 42 mujeres, que cursaban la carrera de Biología y de Licenciatura en Ciencias Naturales y Educación Ambiental en una universidad pública colombiana. Los participantes se dividieron en cuatro grupos equitativos, de acuerdo a la carrera y a la condición de haber cursado o no durante el 2018 dos asignaturas obligatorias del currículo relacionadas con la ecología. El objetivo de las asignaturas fue desarrollar competencias teóricas para: a) establecer el funcionamiento y estructura de los ecosistemas en términos energéticos y poblacionales, sus tipos y principales problemas ambientales que los afectan; b) determinar la multicausalidad de los problemas ambientales desde lo local hasta lo global; c) identificar la importancia de la gestión ambiental en la toma de decisiones para la mitigación, prevención y eliminación de impactos ambientales, mediante la ubicación del papel real de las organizaciones humanas en la perdida y desequilibrio de los ecosistemas. En esta medida, cursar o no las asignaturas debería diferenciar los grupos por nivel de experticia en el dominio específico.

\section{Herramientas}

Entrevista semiestructurada. Conformada por 19 preguntas (ver Apéndice 1), de aplicación individual, con duración de 20 a 40 minutos, para identificar la presencia o ausencia de elementos del modelo ECF, relacionados con el efecto de las actividades antrópicas sobre los ecosistemas (e.g., residuos, tipos de contaminantes, daños a los ecosistemas). La validez de contenido fue determinada por dos biólogos expertos y un experto licenciado en biología ( Kappa $C C I=0,91$ ), se realizaron los ajustes requeridos en cuanto a redacción y contenido.

Situación de Resolución de Problema (SRP) "EI Mariposario". Con el método del análisis de tarea (Pascual-Leone \& Johnson, 1991) se diseñó una SRP (ver Apéndice 2) para rastrear cómo los estudiantes universitarios resuelven la alteración del equilibrio dinámico de un ecosistema, siguiendo el modelo ECF para sistemas complejos (Goel, et al. 1996; Hmelo \& Green, 2004). Siguiendo a Ávila y Triviño (2019), la SRP fue ambientada en un macro proyecto de conservación ambiental y así llamar la atención sobre la problemática de los páramos en Colombia que han sido transformados por la actividad antrópica generando la pérdida y transformación del hábitat de los lepidópteros. La aplicación de la SRPfue individual (duración de 15 a 30 minutos) y se realizó una aplicación piloto previa con un grupo de 10 estudiantes (diferentes a los participantes de la muestra). 


\section{Procedimiento}

Para la recolección de los datos fueron entrenados cuatro psicólogos. En primer lugar, en ambiente controlado de ruido, se realizó la entrevista semiestructurada, la cual fue audio grabada, transcrita textualmente, segmentada por línea y codificada cuantitativamente siguiendo la tabla 1 . En un segundo lugar, para la SRP, los escritos de los estudiantes fueron segmentados por respuestas y se determinó el nivel de desempeño siguiendo la tabla 2. Usando el SPSS 28, se realizaron análisis descriptivos no paramétricos, de acuerdo a las características de las variables. A nivel cualitativo, se categorizaron las explicaciones prototípicas del funcionamiento de la dinámica ecosistémica y sus sesgos, a partir de los conceptos claves y sus relaciones e interdependencias obtenidas de los análisis y segmentaciones de los discursos de los participantes. El resultado de este análisis se observa en las figuras 1 y 2 , en las cuales se construyeron los modelos conceptuales que dan cuenta de cómo lograr comprender el funcionamiento de un sistema complejo.

Esta investigación contó con el aval del Comité Curricular del Programa de Psicología de la Universidad Pedagógica y Tecnológica de Colombia y, en su ejecución, se salvaguardaron todos los principios éticos y normas legales que rigen la investigación en Psicología. Cada participante fue informado sobre el propósito de la investigación, la duración y procedimientos, el carácter voluntario de la participación, las formas de codificación para garantizar el anonimato y la confidencialidad de la información y firmó el consentimiento para participar.

Tabla1.

Componentes del funcionamiento ecosistémico a partir del modelo ECF.

\begin{tabular}{|c|c|c|}
\hline Componente & Indicador & $\begin{array}{l}\text { Ejemplos de la transcripción de } \\
\text { datos }\end{array}$ \\
\hline Estructura & $\begin{array}{c}\text { Presencia o ausencia de } \\
\text { elementos estructurales del } \\
\text { sistema (e.g. plantas, animales, } \\
\text { agua) }\end{array}$ & $\begin{array}{l}\text { "en el interior de un bioterio se puede } \\
\text { encontrar la especie a estudiar, plantas } \\
\text { ehh factores como la temperatura, el } \\
\text { aire, la luz solar" } \\
\end{array}$ \\
\hline Comportamiento & $\begin{array}{l}\text { Presencia o ausencia de } \\
\text { interacciones o mecanismos que } \\
\text { informan de un producto, reacción } \\
\text { o un resultado (e.g., las plantas } \\
\text { trasforman la energía solar en } \\
\text { energía química a través de la } \\
\text { fotosíntesis) }\end{array}$ & $\begin{array}{l}\text { "a través de la sábila podemos obtener } \\
\text { ciertos azucares a través del proceso } \\
\text { de fotosíntesis, la energía nos la va a } \\
\text { proporcionar el sol, quien es quien } \\
\text { activa los cloroplastos de la plantas } \\
\text { para la fotosíntesis, el CO2 pueden ser } \\
\text { de los desechos de los procesos de } \\
\text { respiración de los insectos, el agua la } \\
\text { podemos encontrar en diversos } \\
\text { estados, siendo el estado líquido el que } \\
\text { nos proporciona el adecuado desarrollo } \\
\text { para generar la glucosa" }\end{array}$ \\
\hline Función & $\begin{array}{l}\text { Presencia o ausencia del rol que } \\
\text { cumplen ciertos elementos dentro } \\
\text { del sistema (e.g. las plantas }\end{array}$ & $\begin{array}{l}\text { "las plantas hospederas que permite } \\
\text { que las mariposas depositen su huevos } \\
\text { y las larvas se puedan alimentar de eso }\end{array}$ \\
\hline
\end{tabular}


necesitan luz para realizar la debe tener las fuentes de alimento" fotosíntesis)

Nota: Adaptado de Hmelo \& Green (2004)

Tabla 2. Niveles de desempeño ante la situación de solución del problema "el mariposario".

\begin{tabular}{|c|c|c|}
\hline Desempeño & Descripción & Ejemplo de la transcripción de datos \\
\hline Muy Bajo & $\begin{array}{l}\text { Enuncia de forma fragmentada o } \\
\text { desarticulada causas de equilibrio } \\
\text { o desequilibrio del ecosistema } \\
\text { asociadas a la carencia de } \\
\text { elementos estructurales o a una } \\
\text { función errónea. } \\
\end{array}$ & $\begin{array}{c}\text { "el desabastecimiento alimentario se dio } \\
\text { debido a que faltaba información acerca de } \\
\text { estos lepidópteros y por lo tanto al llevar este } \\
\text { proyecto acabo salieron a flote estas } \\
\text { falencias" }\end{array}$ \\
\hline Bajo & $\begin{array}{l}\text { Establece relación causal directa } \\
\text { entre al menos un elemento } \\
\text { estructural del desequilibrio del } \\
\text { ecosistema y un elemento } \\
\text { comportamental consecuente o } \\
\text { una función derivada de la causa. }\end{array}$ & $\begin{array}{l}\text { "el desabastecimiento de alimento para las } \\
\text { lepidópteras en estado larval y adulto, se } \\
\text { presenta por la falta de plantas que } \\
\text { proporcionan las condiciones óptimas para el } \\
\text { desarrollo de la especie" }\end{array}$ \\
\hline Medio & $\begin{array}{l}\text { Establece una cadena causal entre } \\
\text { al menos dos elementos } \\
\text { estructurales, un comportamiento } \\
\text { y una función que logran explicar } \\
\text { el desequilibrio del ecosistema. }\end{array}$ & $\begin{array}{l}\text { "los recursos usados por larvas y adultos } \\
\text { (plantas) no están completando su ciclo, } \\
\text { debido al encierro se puede estar cortando } \\
\text { procesos ecosistémicos claves como la } \\
\text { dispersión, probablemente se aislaron los } \\
\text { polinizadores que permitían la reproducción } \\
\text { de las plantas, al no haber polinización no hay } \\
\text { reproducción y por tanto no se está } \\
\text { regenerando la población de plantas" }\end{array}$ \\
\hline Alto & $\begin{array}{c}\text { Establece una cadena } \\
\text { multicausales entre los diferentes } \\
\text { elementos estructurales, } \\
\text { comportamientos y funciones que } \\
\text { logran explicar el desequilibrio del } \\
\text { ecosistema. Esta puntuación } \\
\text { incluye, las relaciones causales } \\
\text { mediadas entre los componentes } \\
\text { del modelo ECF. }\end{array}$ & $\begin{array}{c}\text { "La espeletiamiradorensis (Frailejon) y } \\
\text { chusqueatesellata son plantas endémicas } \\
\text { paramunas, las cuales requieren de } \\
\text { regímenes hídricos específicos para el } \\
\text { mantenimiento y desarrollo biológico correcto. } \\
\text { Al someterse a presiones externas de estrés, } \\
\text { como disminución de agua, van a empezar a } \\
\text { sufrir cambios fisiológicos que pueden } \\
\text { ocasionar la muerte de las especies de } \\
\text { plantas" }\end{array}$ \\
\hline
\end{tabular}

Nota: Fuente elaboración propia.

\section{Resultados}

\section{Entrevista semiestructurada}

Teniendo en cuenta el modelo ECF, la Tabla 3 muestra que los conceptos más usados por los estudiantes fueron: plantas para la estructura; transformación de la energía para el comportamiento; y las plantas como fuente de alimento para la función. El uso espontáneo de estos conceptos indicó que los estudiantes poseen el conocimiento previo para abordar la tarea planteada en este estudio, independientemente de la carrera 
cursada. No obstante, aquellos estudiantes que cursaron las dos asignaturas relacionadas con la ecología logran articular mayor número de conceptos claves del modelo ECF.

Tabla 3.

Frecuencia de uso de conceptos ecológicos durante la entrevista semiestructurada.

\begin{tabular}{|c|c|c|c|c|c|}
\hline Estructura & $\begin{array}{l}\text { Frec. } \\
(\%)\end{array}$ & Comportamiento & Frec.(\%) & Función & Frec. (\%) \\
\hline Plantas & $55(23,4)$ & $\begin{array}{c}\text { Transformación de } \\
\text { la eneraía }\end{array}$ & $55(18,2)$ & Especie indicadora & $19(9,8)$ \\
\hline Mariposa & $55(23,4)$ & $\begin{array}{c}\text { Ciclos } \\
\text { biogeoquímicos }\end{array}$ & $55(18,2)$ & $\begin{array}{l}\text { La mariposa como } \\
\text { polinizador y agente } \\
\text { de dispersión de } \\
\text { polen }\end{array}$ & $38(19,6)$ \\
\hline Luz & $34(14,5)$ & $\begin{array}{l}\text { Procesos de flujo } \\
\text { de energía }\end{array}$ & $55(18,2)$ & $\begin{array}{l}\text { Las plantas como } \\
\text { fuentes de alimento } \\
\text { y refugio }\end{array}$ & $49(25,3)$ \\
\hline Agua & $54(23)$ & $\begin{array}{l}\text { Procesos } \\
\text { reproductivos }\end{array}$ & $48(15,8)$ & $\begin{array}{c}\text { Plantas como } \\
\text { purificadores de aire } \\
\text { y agua }\end{array}$ & $19(9,8)$ \\
\hline \multirow[t]{2}{*}{ Humedad } & $37(15,7)$ & $\begin{array}{l}\text { Proceso de } \\
\text { fotosíntesis }\end{array}$ & $47(15,5)$ & $\begin{array}{l}\text { Plantas como } \\
\text { productores de } \\
\text { oxigeno }\end{array}$ & $27(13,9)$ \\
\hline & & $\begin{array}{c}\text { Relación simbiótica } \\
\text { entre la mariposa y } \\
\text { las plantas }\end{array}$ & $42(13,9)$ & $\begin{array}{l}\text { Factores abióticos } \\
\text { como elementos } \\
\text { indispensables para } \\
\text { recrear el hábitat }\end{array}$ & $41(22,1)$ \\
\hline Total & $235(100)$ & Total & 403 & Total & 193 \\
\hline
\end{tabular}

El análisis cuantitativo del uso de los conceptos reveló diferencias estadísticamente significativas entre los estudiantes de biología y de licenciatura en el componente estructura $\left(U_{(\mathrm{n}=60)}=265,500, z=-2,731, p=0,006\right)$ y en el componente función $\left(U_{(\mathrm{n}=60)}=\right.$ 287,000, $z=-2,080, p=0,038$ ). Los rangos promedios de las medianas de los estudiantes de biología fueron más altos en conceptos relacionados con la estructura del sistema (Biología $M d=36,65$; Licenciatura $M d=24,65$ ) y su función (Biología $M d=34,10$; Licenciatura $M d=24.90)$. En el componente comportamiento los estudiantes de biología los rangos promedio fueron más altos, pero sin diferencias estadísticas $(p>0,05)$ (Biología $M d=32,29$; Licenciatura $M=26,82$ ).

En la Figura 1 y 2 se diagrama el modelo conceptual construido por los estudiantes. Las figuras ilustran el uso de los conceptos claves y las relaciones e interdependencias identificadas en el análisis del discurso de los participantes.En ambas carreras se privilegiaron los elementos estructurales con saliencia perceptual en un bioterio. Sin embargo, los estudiantes de Biología integraron más conceptos claves estructurales (e.g., cantidad de plantas hospederas y nutricias, cantidad de alimento, materia inorgánica, factores abióticos tales como el agua, la luz, la temperatura y la humedad), en comparación de los estudiantes de Licenciatura (e.g., presencia de plantas, mariposa y rocas, necromasa y factores abióticos como la luz, el agua, clima). Los estudiantes de 
Biología establecieron las interrelaciones y multicausalidades(observar líneas dobles y segmentadas en la figura 1) entre las estructuras y las funciones del sistema para mantener el equilibrio del bioterio (e.g., la planta genera alimento con glucosa y oxígeno para la supervivencia de la mariposa; reproducción de la planta por la polinización que efectúa la mariposa; degradación de la materia).

A partir de la relación entre las estructuras y las funciones, ellos infirieron los comportamientos frente al flujo de energía y la relación existente entre los procesos respiratorios de las plantas y las mariposas (i.e., relación entre la planta, la mariposa y el ciclo del carbono, la transformación de la energía). En contraste, los estudiantes de Licenciatura enunciaron elementos e interrelaciones entre las estructuras, los comportamientos y las funciones del sistema, pero, estos no incluyeron conceptos claves o descripciones de los procesos subyacentes para mantener el equilibrio del bioterio (e.g., el ciclo del carbono, la transformación de energía). Los estudiantes de licenciatura incluyeron el clima como un factor abiótico estructural, lo cual construye un sesgo conceptual porque este es un comportamiento producto de la interacción de varios factores abióticos.

Figura 1.

Modelo conceptual de estudiantes de Biología $(n=30)$

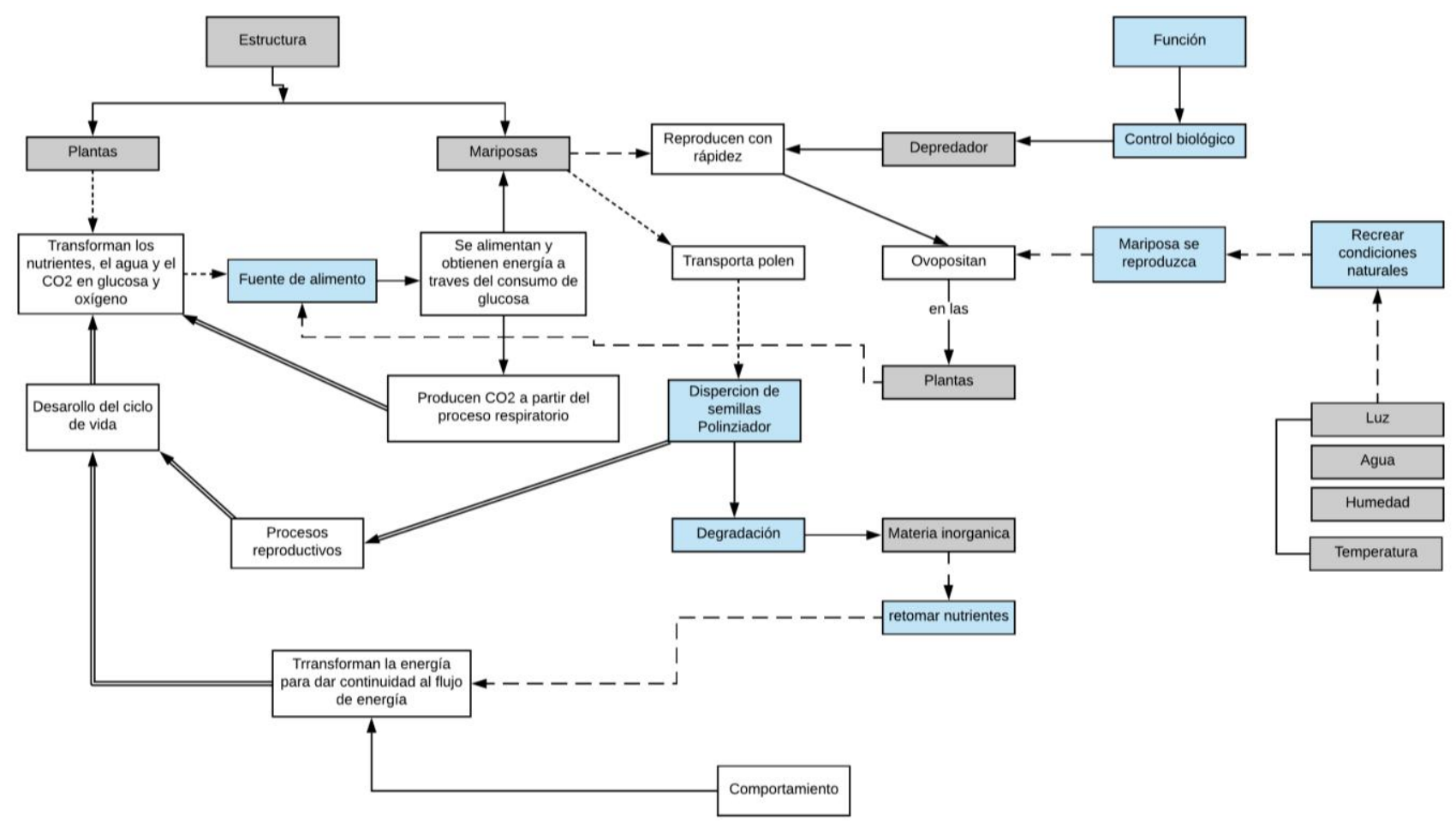

Nota: Fuente elaboración propia. Líneas dobles representan procesos multicausales; líneas segmentadas representan cadenas causales tipo E-F-C; líneas puntuadas representan cadenas de tipo E-C-F 
Figura 2.

Modelo conceptual de estudiantes de Licenciatura en Ciencias Naturales y Educación Ambiental $(n=30)$

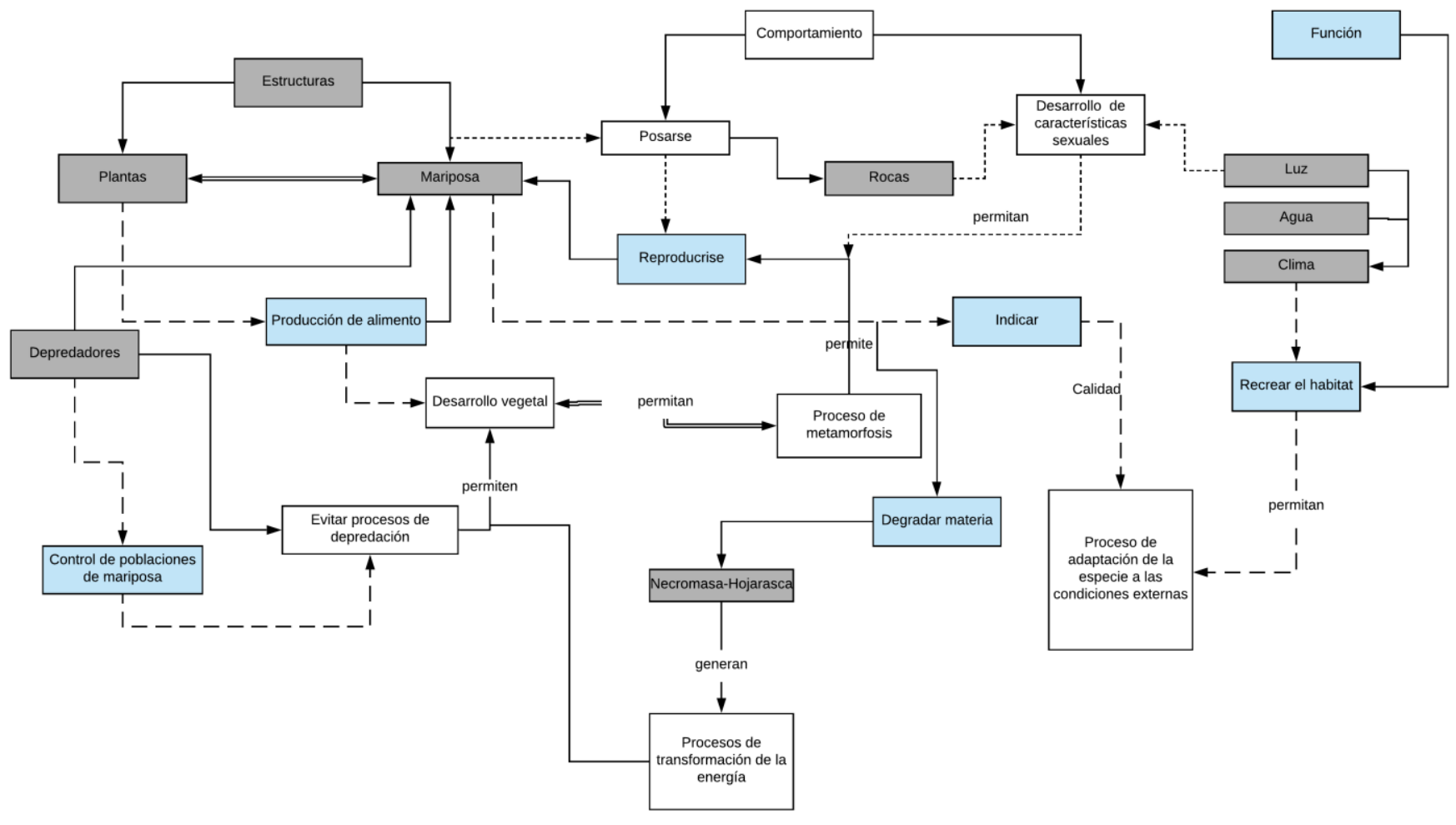

Nota: Fuente elaboración propia. Líneas dobles representan procesos multicausales; líneas segmentadas representan cadenas causales tipo E-F-C; líneas puntuadas representan cadenas de tipo E-C-F

La prueba U de Mann Whitney mostró diferencias estadísticamente significativas entre los modelos conceptuales de los estudiantes que cursaron y no cursaron la asignatura ecología en el componente comportamiento del sistema $\left(U_{(\mathrm{n}=60)}=294,500, z=-2,135, p\right.$ $=0,033)$. Quienes cursaron la asignatura obtuvieron medianas con rangos promedio superiores $(M d=34,68)$, revelando la incorporación de comportamientos relacionados a los procesos de flujo de energía (e.g., la descomposición de la materia, la fotosíntesis y la respiración vegetal y animal), los procesos de polinización (e.g. la relación interdependiente que tienen la mariposa con la planta nutricia que les permite a ambos individuos desarrollarse), la absorción y la producción de minerales en el suelo del bioterio (e.g. los procesos mediante los cuales se produce la degradación de la materia orgánica y cómo este proceso permite la recirculación de la energía). Por su parte, quienes no cursaron las asignaturas de ecología $(M d=25,16)$ privilegiaron la interacción de comportamientos relacionados con los procesos respiratorios de la planta y la mariposa, omitiendo la relación con otros procesos de mayor relevancia ecosistémica, por ejemplo, la fotosíntesis. 
Por su parte, la prueba Kruskall Wallis reveló diferencias estadísticamente significativas entre los cuatro grupos comparados: en el componente estructura $\left(X_{(3, \mathrm{n}=60)}^{2}=8,760, p=\right.$ $0,033)$ y en el componente comportamiento $\left(X_{(3, \mathrm{n}=60)}^{2}=8,042, p=0,045\right)$ (Ver tabla 4). Lo que esto indica es que, los estudiantes que cursaron ecología lograron establecer inferencias deductivas sobre aquellas relaciones que subyacen a los procesos del funcionamiento del bioterio como producto de las interacciones entre los comportamientos y las funciones de las estructuras identificadas en la explicación.

Tabla 4.

Distribución de rango promedio de la comprensión del funcionamiento ecosistémico por haber cursado o no las asignaturas de ecología.

\begin{tabular}{|c|c|c|c|c|c|c|}
\hline Modelo & Grupos & $\boldsymbol{N}$ & $\begin{array}{c}\text { Rango } \\
\text { promedio }\end{array}$ & $x^{2}$ & gl & $\boldsymbol{p}$ \\
\hline \multirow{5}{*}{ Estructura } & Biología si cursaron ecología & 15 & 34,83 & & & \\
\hline & Biología no cursaron ecología & 15 & 38,47 & & & \\
\hline & Licenciatura si cursaron ecología & 15 & 27,50 & & & \\
\hline & Licenciatura no cursaron ecología & 15 & 21,20 & & & \\
\hline & Total & 60 & & 8,760 & 3 & 0,033 \\
\hline \multirow{5}{*}{$\begin{array}{l}\text { Comportam } \\
\text { iento }\end{array}$} & Biología si cursaron ecología & 15 & 35,17 & & & \\
\hline & Biología no cursaron ecología & 14 & 31,29 & & & \\
\hline & Licenciatura si cursaron ecología & 15 & 34,20 & & & \\
\hline & Licenciatura no cursaron ecología & 15 & 19,43 & & & \\
\hline & Total & 59 & & 8,042 & 3 & 0,045 \\
\hline \multirow{5}{*}{ Función } & Biología si cursaron ecología & 15 & 36,30 & & & \\
\hline & Biología no cursaron ecología & 14 & 31,75 & & & \\
\hline & Licenciatura si cursaron ecología & 14 & 25,46 & & & \\
\hline & Licenciatura no cursaron ecología & 15 & 24,37 & & & \\
\hline & Total & 58 & & 4,883 & 3 & 0,181 \\
\hline
\end{tabular}

Nota: Elaboración propia.

\section{Solución de problema en la tarea propuesta}

El análisis Chi Cuadrado indicó que existe independencia $(p>0,05)$ entre el nivel de sofisticación de la solución a la tarea y la carrera cursada, para quienes han cursado o no las dos asignaturas de ecología. El 83\% de los estudiantes lograron resolver la SRP, con diferentes niveles de sofisticación. En la Tabla 5 se observa que son más las distribuciones de estudiantes que se ubicaron en niveles de desempeño bajo $(n=21)$ y medio $(n=21)$, lo cual indica que reconocieron y articularon uno o dos elementos del sistema respectivamente, así como sus interrelaciones, para razonar sobre el impacto de las actividades antropogénicas (e.g. contaminación) sobre el funcionamiento de un ecosistema en un bioterio para mariposas. Los estudiantes con niveles de desempeño bajo y medio en la SRP, construyeron sesgos causales en sus explicaciones porque no reconocieron la multicausalidad de la contaminación de las fuentes hídricas que abastecían el mariposario recreado en la tarea y tampoco describieron cómo esto guardaba relación con la disponibilidad de alimento de la mariposa para sobrevivir. 
Los estudiantes que se ubicaron en el nivel alto $(n=8)$, de mayor grado de sofisticación, articularon en razonamiento la multicausalidad de la contaminación de las fuentes hídricas, así como la relación entre la escasez de alimentos, la cual ha sido efecto de la contaminación y el proceso de diapausa en el que los individuos entran al no obtener recursos suficientes (e.g., disminución de su tasa metabólica y disminución del comportamiento reproductivo). Este subgrupo de estudiantes, generalmente, también articularon soluciones al problema de la contaminación y la escasez de alimentos relacionadas con implementar modelos de siembra escalonada y descontaminación del agua, lo cual revela la articulación entre los componentes del modelo ECF. Esto es, a nivel de estructura, este subgrupo de estudiantes reconoció el agua y la cantidad de plantas en relación a la función que esta estructura cumplió en el ciclo vital de las mariposas. A nivel del comportamiento, ellos reconocieron la interdependencia de la calidad del agua, el proceso de fotosíntesis de la planta y la glucosa disponible como alimento para las mariposas, al mismo tiempo que, identificar cómo esto soporta los procesos de reproducción y sobrevivencia de la especie. $Y$, a nivel del funcionamiento, ellos reconocieron la relación simbiótica entre las plantas y las mariposas, es decir, que estructuralmente las mariposas polinizaron las plantas y, a su vez, las plantas refugiaron a las mariposas y las abastecieron de alimento durante el proceso de la metamorfosis, garantizando la reproducción de ambas especies.

Finalmente, los estudiantes que se ubicaron en el nivel más bajo de desempeño $(n=10)$, generalmente, razonaron basados en elementos estructurales sin ninguna articulación con el comportamiento o la función. Por ejemplo, construyeron como solución trasladar el bioterio de lugar para mejorar las condiciones de control y equilibrio que el biólogo puede tener sobre el sistema. Este tipo de explicaciones no consideró que, el carácter endémico de la especie y que los factores abióticos del hábitat son esenciales para la reproducción de las mariposas (lymanopodamirabilis) y las plantas (i.e., frailejon, chusqueatessellota), de acuerdo a la etapa de la metamorfosis (i.e., larva, pupa, imago).

Tabla 8.

Distribución de nivel de desempeño obtenido ante la situación de resolución de problemas

\begin{tabular}{|c|c|c|c|c|c|c|c|}
\hline \multirow[t]{2}{*}{ Carrera } & \multicolumn{4}{|c|}{$\begin{array}{c}\text { Nivel de Desempeño frente situación } \\
\text { problema Frec.(\%) }\end{array}$} & \multirow[b]{2}{*}{$x^{2^{*}}$} & \multirow[b]{2}{*}{ gl } & \multirow[b]{2}{*}{$p$} \\
\hline & Muy bajo & Bajo & Medio & Alto & & & \\
\hline $\begin{array}{l}\text { Biología - Cursaron } \\
\text { ecología }\end{array}$ & $1(10)$ & $7(33,3)$ & $5(23,8)$ & $2(25)$ & & & \\
\hline $\begin{array}{c}\text { Biología - No cursaron } \\
\text { ecología }\end{array}$ & $1(10)$ & $4(19)$ & $7(33,3)$ & $3(37,5)$ & & & \\
\hline $\begin{array}{l}\text { Licenciatura - Cursaron } \\
\text { ecología }\end{array}$ & $5(50)$ & $3(14,3)$ & $6(28,6)$ & $1(12,5)$ & & & \\
\hline $\begin{array}{l}\text { Licenciatura - No } \\
\text { cursaron ecología }\end{array}$ & $3(30)$ & $7(33,3)$ & $3(14,3)$ & $2(25)$ & & & \\
\hline Total & $10(100)$ & $21(100)$ & $21(100)$ & $8(100)$ & 9,493 & 8 & 0,393 \\
\hline
\end{tabular}

Nota: Fuente elaboración propia. 


\section{Discusiones y conclusiones}

Los hallazgos de esta investigación revelaron que, la mayoría de los estudiantes universitarios comprenden el funcionamiento complejo de un ecosistema, de acuerdo al modelo ECF planteado por Hmelo y Green (2004). Los participantes de los cuatro grupos, generalmente, iniciaron su razonamiento desde el reconocimiento de los elementos estructurales con mayor saliencia perceptual en el funcionamiento de un ecosistema y de un bioterio, para luego, articular relaciones causales con las funciones y el comportamiento del sistema complejo. Hmelo y Green (2004) argumentan que, las estructuras son el nivel más privilegiado cognitivamente por los estudiantes principiantes en un dominio disciplinar, debido a la disponibilidad cognitiva, siendo el elemento sin el cual el razonamiento sobre el funcionamiento del sistema complejo no se genera.

Entre los grupos participantes se identifican diferencias en el razonamiento, atribuibles al nivel de conocimiento o experticia. Los estudiantes de biología que cursaron ecología, seguidos por los de licenciatura que cursaron ecología, incorporan la interacción cadenas causales E-F-C o E-C-F, así como la interacción simultánea entre diferentes agentes del ecosistema en el nivel estructural. Además, ellos indican información sobre los mecanismos funcionales en la dinámica ecosistémica (e.g., el papel de la fotosíntesis en la producción de alimento necesario para el desarrollo metamórfico de la mariposa), al mismo tiempo que, ellos incorporan conocimiento previo disciplinar no explícito en la entrevista o en la tarea (e.g., la polinización o los procesos de transformación materia y energía). De esta forma, cuando los estudiantes incorporan la articulación multicausal existente entre los diferentes componentes y niveles del sistema, ellos logran explicar de forma plausible el funcionamiento del ecosistema, a pesar de no incluir todos los elementos posibles.

Sánchez y Pontes (2010) argumentan la necesidad de conocer las ideas previas de los estudiantes al iniciar las asignaturas para, desde allí, el profesor logre orientar las estrategias de desarrollo de las habilidades y competencias cognitivas necesarias para la comprensión de fenómenos emergentes complejos. Los profesores universitarios encargados de formar a los futuros biólogos y licenciados (quienes en el futuro también enseñaran en las escuelas y universidades), deberían diseñar estrategias que permitan conocer cuáles son los sesgos en los procesos de razonamiento y dedicar parte su proceso formativo a que estos sean resueltos. El aula de clase es un espacio propicio para generar cambios en los procesos de construcción de conocimiento y usar el modelo ECF es una vía viable para desarrollar las competencias cognitiva que posibilitan en el estudiante estructurar el conocimiento nuevo que construye.

En este estudio se identifican cinco sesgos en el razonamiento sobre la causalidad en los sistemas complejos. En primer lugar, ante la exigencia cognitiva de establecer la causa posible del desequilibrio del sistema desde la articulación de los tres elementos modelo ECF. Los tres sesgos prototipos son: a) estipular la causa como la ausencia de algún 
elemento estructural (e.g., poca cantidad de plantas); b) identificar como causa el mal funcionamiento de un elemento estructural (e.g., dificultad de las plantas para producir suficiente alimento); y c) enunciar la causa estructural, sin identificar por qué altera la dinámica y el equilibrio del sistema (e.g., enuncian la carencia de alimento para la mariposa en el bioterio, pero no articulan funcional ni causalmente la alimentación de la mariposa, su reproducción y la degradación por procesos químicos) -resultados en la misma línea se encuentran en Kevin y Munson (2008) y Tekkaya (2002).

En segundo lugar, ante la exigencia cognitiva de identificar la relación multicausal entre los diferentes elementos del modelo ECF, bien sea, cadenas causales (e.g., E-F-C; E-C-F) o causalidad mediada (e.g., E-F/E-C-F; E-C/E-F-C) de mayor sofisticación, se construyen sesgos en el razonamiento al establecer la causalidad directa entre un antecedente y un consecuente. Los dos sesgos prototipos restantes son: a) causalidad directa E-F (e.g., "la carencia de agua impide que esta pueda brindar a las plantas los nutrientes suficientes para su adecuado funcionamiento"; "Las mariposas no pueden reproducirse debido a que las plantas no producen suficiente alimento o refugio para las larvas"); y b) Causalidad directa entre E-C (e.g., "Las plantas están realizando procesos biológicos incompletos que afectan los procesos ecosistémicos que impiden la proliferación de plantas").

Resultados similares argumentan Hashem y Mioduser (2013), quienes usaron el modelo ECF en ambientes modelados. Los autores concluyeron que, los estudiantes sesgan la comprensión cuando no comprenden la causalidad y el azar con el que se rige el sistema complejo de carácter multinivel. El tipo de tareas a la que los estudiantes se enfrentan, generan desafíos cognitivos que les permiten comprender las dinámicas funcionales y causales de los sistemas complejos. Por ello, los autores sugieren orientar experiencias de aprendizaje interdisciplinario que promuevan el análisis de patrones, modelos de vinculación de causas locales y comportamiento global, así como el pensamiento sistémico y pensamiento descentralizado. Los hallazgos del presente estudio permiten sugerir los escenarios basados en preguntas y basados en problemas en formatos impresos, para quienes por limitaciones logísticas no pueden generar ambientes simulados o modelados en computadora, como un medio efectivo para incentivar el razonamiento causal y funcional de un sistema complejo. En estos escenarios el modelo ECF puede ser usado como una vía para orientar la estructuración del conocimiento impartido en el aula y posibilitar así el desarrollo cognitivo de los estudiantes.

La evidencia empírica muestra relación entre la buena comprensión de los conceptos que enseñan a los futuros maestros de Biología y su eficacia en el proceso de enseñanza (Buethe \& Smallwood, 1987; Moseley, Reinke \& Bookout, 2003; Mosothwane, 2002; Summers, Kruger \& Childs, 2001). Es fundamental que, la enseñanza de las ciencias, dirigidas a quienes se forman para ser futuros docentes, continúe asumiendo el reto de abordar la comprensión de los sistemas con funcionamiento complejo en diferentes campos y disciplinas, como una forma de favorecer la comprensión causal compleja de las relaciones entre los diferentes componentes de un sistema (e.g., procesos de la biología o 
la física, los ambientes simulados de aprendizaje, la sobrepoblación o el hacinamiento, el altruismo, los fenómenos socio históricos, entre otros) (Centola, Wilensky, \& McKenzie, 2013, Johnson, 2009; Jacobson \& Wilensky, 2006; Navarro Roldán \& Castellanos-Páez, 2017; Wilensky \& Jacobson, 2014).

A partir de las limitaciones de este estudio, se sugieren futuras investigaciones aplicadas que incluyan nuestros probabilísticos, con mayor número de muestra, usando modelos de medición longitudinal para determinar las varianzas en el proceso de comprensión de los estudiantes antes, durante y después de los cursos de ecología, incluyendo procesos de simulación que permitan al estudiante experimentar con varias SRP en las que se puedan controlar los parámetros del ecosistema y analizar cómo el estudiante incorpora la evidencia en el proceso de evaluación de hipótesis que somete a verificación. Este tipo de diseños plantea el reto de proponer escenarios de investigación que permitan identificar las trayectorias epistémicas en los procesos de construcción de los modelos mentales en estudiantes universitarios, así como analizar sus variabilidades. Al mismo tiempo, se sugiere conducir estudios que evalúen la relación entre los procesos emocionales y los procesos de razonamiento frente a la complejidad en dominios de las ciencias naturales y las ciencias sociales. Esto permitirá aportar insumos sobre cómo los procesos emocionales y cognitivos favorecen los procesos de construcción conocimiento en escenarios que demandan y desafían cognitivamente a los estudiantes universitarios, así como demandan procesos que involucran razonamiento moral prosocial, atribuciones causales, empatía u otros predictores de comportamiento social.

\section{Referencias}

Astudillo, H., y Gene , A. (1984). Errores Conceptuales en Biología. La Fotosíntesis de las plantas verdes. Investigación y Experiencias Didacticas, (1), 15-16. https://ddd.uab.cat/pub/edlc/02124521v2n1/02124521v2n1p15.pdf

Ávilar, L., y Triviño, P. (2019). Descripción del hábitat de cuatro especies del género lymanopoda (lepidoptera: Nymphalidae: Satyrinae) en áreas de páramo de Colombia. Acta Biológica Colombiana, 24(1), 125-138. http://dx.doi.org/10.15446/abc.v24n1.70285

Bahar, M. (2003). Misconceptions in Biology Education and Conceptual Change Strategies. Education, Science Theory \& Practice, 3(1), 55-64.

Buethe, C., \& Smallwood, J. (1987). Teachers' Environmental literacy: Check and Recheck, 1975 and 1985. The Journal of Environmental Education, 18(3), 39-42. http://dx.doi.org/10.1080/00958964.1987.9942738

Centola, D., Wilensky, U., \& McKenzie, E. (2000). A Hands-On Modeling Approach to Evolution: Learning about the Evolution of Cooperation and Altruism Through Multi-Agent Modeling - The EACH Project. In B. Fishman \& S. O'Connor-Divelbiss 
(Eds.), Fourth International Conference of the Learning Sciences (pp. 166-173). Mahwah, N]: Erlbaum. https://pdfs.semanticscholar.org/2663/81836b1e5259ca0d99e0b0cabf96801a5c19. pdf

Chi, T., Slotta, J., \& De Leeuw, N. (1994). From things to processes: A theory of conceptual change for learning science concepts. Learning and Instruction, 4(1), 27-43. http://dx.doi.org/10.1016/0959-4752(94)90017-5

Chi, T. (2005). Commonsense conceptions of emergent processes: Why some misconceptions are robust. The journal of the learning sciences, 14(2), 161-199. http://dx.doi.org/10.1207/s15327809jls1402_1

Chi, M. T., Roscoe, R. D., Slotta, J. D., Roy, M., \& Chase, C. C. (2012). Misconceived Causal Explanations for Emergent Processes. Cognitive Science, 36(1), 1-61. http://dx.doi.org/10.1111/j.1551-6709.2011.01207.x

Chung, H, T., Brazis, P., Rocci, M., Kannewurf, C., Bastea, M., \& Uher, C. (2000). CsBi4Te6: A High-Performance Thermoelectric Material for Low-Temperature Applications. Science, 287(5455), 1024-1027. http://dx.doi.org/10.1126/science.287.5455.1024

D'Avanzo, C. (2003). Application of research on learning to college teaching: ecological examples. Bioscience, 53(11), 1121-1128. http://dx.doi.org/10.1641/00063568(2003)053[1121:AOROLT]2.0.CO;2

Eyster, L. S., \& Tashiro , J. S. (1997). Using manipulatives to teach quantitative concepts in ecology.A Hands-On Method for Detecting \& Correcting Misconceptions about Limiting Factors in Eutrophication \& Vegetarianism. American Biology Teacher, 59(6), 360 364. http://dx.doi.org/10.2307/4450330

Ferrari, M. \& Chi, M. (1998). The nature of naive explanations of natural selection. International Journal of ScienceEducation, 20(10), 1231-1256, http://dx.doi.org/10.1080/0950069980201005

García, R. (2011) Interdisciplinariedad y sistemas complejos. Revista Latinoamericana de Metodología de las Ciencias Sociales, $1, \quad(1), \quad 66-101$. http://www.memoria.fahce.unlp.edu.ar/art_revistas/pr.4828/pr.4828.pdf

García, R. (2006). Sistemas complejos Conceptos, método y fundamentación epistemológica de la investigación interdisciplinaria. https://docs.ufpr.br/ edugeo/GB082/Bibliografia/Garcia_Sistemas\%20Complejos.p df

Goel, A. K., Gómez de Silva Garza, A., Grué, N., Murdock, J.W., Recker,M. M., \& Govinderaj, T. (1996). Towards designing learning environments. I: Exploring how devices work. Lecture Notes in Computer Science, 493-501. http://dx.doi.org/10.1007/3-540-61327-7_148 
Hashem, K., \& Mioduser, D. (2013). Learning by Modeling (LbM): Understanding Complex Systems by Articulating Structures, Behaviors, and Functions. International Journal of Advanced Computer Science and Applications, 4(4), 80-86. http://dx.doi.org/10.14569/ijacsa.2013.040414

Hernández-Sampieri, R., Fernández, C., y Baptista, P. (2010). Metodología de la investigación. México: McGraw-Hill Interamericana.

Hmelo, C. E., \& Green, M. (2004). Comparing expert and novice understanding of a complex system from the perspective of structures, behaviors, and functions. Cognitive Science, 28(1), 127-138. http://dx.doi.org/10.1016/s03640213(03)00065-x

Hmelo, C. E., Holton, D. L., \& Kolodner, J. L. (2000). Designing to Learn About Complex Systems. Journal of the Learning Sciences, 9(3), 247-298. http://dx.doi.org/10.1207/s15327809jls0903_2

Hogan, K., \& Fisherkeller, J. (1996). Representing students' thinking about nutrient cycling in ecosystems: Bidimensional coding of a complex topic. Journal of Research in Science Teaching: The Official Journal of the National Association for Research in Science Teaching, 33(9), 941-970. https://doi.org/10.1002/(SICI)10982736(199611)33:9<941::AID-TEA1>3.0.CO;2-V

Hogan, K. (2000). Assessing students' systems reasoning in ecology. Journal of Biological Education, 35(1), 22-28. https://doi.org/10.1080/00219266.2000.9655731

Hungerford, H., \& Volk, T. (1990). Changing learner behavior through environmental education. The Journal of Environmental Education, 21(3), 8-21. http://dx.doi.org/10.1080/00958964.1990.10753743

Jacobson, M. (2001). Problem solving, cognition, and complex systems: Differences between experts. Complexity, 6(3), 41-49. http://dx.doi.org/10.1002/cplx.1027

Jacobson, M., \& Wilensky, U. (2006). Complex systems in education: Scientific and educational importance and implications for the learning sciences. The Journal of the learning sciences, 15(1), 11-34. https://doi.org/10.1207/s15327809jls1501_4

Jeffries, H., Stanisstreet, M., \& Boyes , E. (2001). Knowledge about the 'greenhouse effect': Have college students improved? Research in Science and Technological Education, 19(2), 205-221. http://dx.doi.org/10.1080/02635140120087731

Johnson, N. (2009). Simply complexity: A clear guide to complexity theory. Inglaterra: Oneworld Publications.

Kevin, M., \& Munson , B. H. (2008). An Exploratory Study of Elementary Preservice Teachers Understanding of Ecology Using Concept Maps. The Journal of Environmental Education, 39(3), 32-46. http://dx.doi.org/10.3200/joee.39.3.32-46 
Mahmud, M., \& Gutiérrez, O. (2010). Estrategia de Enseñanza Basada en el Cambio Conceptual para la Transformación de Ideas Previas en el Aprendizaje de las Ciencias. Formación Universitaria, 3(1), 11-20. http://dx.doi.org/10.4067/s071850062010000100003

Moseley, C., Reinke, K., \& Bookout, V. (2003). The effect of teaching outdoor environmental education on elementary preservice teachers' self-efficacy. Journal of Elementary Science Education, 15(1), 1-14. https://doi.org/10.1007/BF03174740

Mosothwane, M. (2002). Pre-Service Teachers' Conceptions of Environmental Education. Research in Education, 68(1), 26-40. http://dx.doi.org/10.7227/rie.68.3

Munson, B. H. (1994). Ecological Misconceptions. The Journal of Environmental Education, 25(4), 30-34. http://dx.doi.org/10.1080/00958964.1994.9941962

Navarro-Roldán, C. P., \& Castellanos-Páez, V. (2017). Cambio conceptual en el aprendizaje escolar de la Historia. CES Psicología, 10(2), 1-16. http://dx.doi.org/10.21615/cesp.10.2.1

Pascual-Leone, J., \& Johnson, J. (1991). The psychological unit and its role in task analysis: A reinterpretation of object permanence. En M. Chandler y M. Chapman (Eds.), Criteria for competence: Controversies in the conceptualization and assessment of children's abilities (pp. 151-187). Lawrence Erlbaum Associates.

Perkins, D. N., \& Grotzer, T. A. (2000). Models and moves: Focusing on dimensions of causal complexity to achieve deeper scientific understanding. Conferencia presentada en Annual Meeting of the American Educational Research Association, New Orleans, LA. https://eric.ed.gov/?id=ED441698

Sánchez, F. J., \& Pontes, A. (2010). La comprensión de conceptos de ecología y sus implicaciones para la educación ambiental. Revista Eureka sobre Enseñanza y Divulgación de las Ciencias, (7), 270-285. http://www.apac-eureka.org/revista

Shepardson, O. P. (2005). Student Ideas: What Is An Environment? Journal of Environmental Education, 36, 39-59. https://search. proquest.com/openview/d7498601bbfa98fb687f0126cc4e996f/1?pqorigsite $=$ gscholar\&cbl $=34493$

Summers, M., Kruger, C., \& Childs, A. (2001). Understanding the science of environmental issues: development of a subject knowledge guide for primary teacher education. International Journal of Science Education, 23(1), 33-53. http://dx.doi.org/10.1080/09500690116990

Tekkaya, C. (2002). Misconceptions as barrier to understanding biology. Hacettepe University Education Journal, 23, 259-266. https://dergipark.org.tr/en/download/article-file/87939 
Weld, D. S. (1983). Explaining complex engineered devices. Inglatera: Cambridge, Bolt Beranek and Newman

Wilensky, U., \& Jacobson, M. J. (2014). Complex systems and the learning sciences. En The Cambridge Handbook of the Learning Sciences (Second Edition; pp. 319338). Cambridge University Press.

Wilensky, U., \& Resnick, M. (1999). Thinking in levels: A dynamic systems approach to makingsense of the world. Journal of Science Education and Technology, 8(3), 319. http://dx.doi.org/10.1023/A:1009421303064 


\section{Apéndice 1}

\section{Entrevista semiestructurada sobre el funcionamiento de un ecosistema en bioterio.}

\section{Parte 1. Identificación de elementos estructurales que componen el sistema}

1. ¿Qué podemos encontrar al interior de un bioterio?

2. ¿Qué factores considera usted que son importantes para el éxito reproductivo de la especie al interior del bioterio?

3. Desde el punto de vista ecológico ¿Qué factores considera usted que son importantes para el éxito funcional del bioterio?

4. Haga un listado tentativo de los elementos para construir el bioterio separando en la categoría biótica y la abiótica

5. Haga un esquema general utilizando la lista que propuso anteriormente indicando el flujo de energía y materia al interior del mismo.

Parte 2. Funcionamiento de las estructuras en el sistema

1. En general, ¿Qué papel cumplen los factores abióticos y bióticos al interior del bioterio?

2. Desde el punto de vista ecológico ¿Qué factores considera usted que son importantes para el éxito funcional?

3. Según el ejemplo propuesto en el formato de la tarea usted está criando mariposas, ¿qué función cumplen estas al interior de un bioterio?

4. Compare las funciones generales que cumplen las plantas en el ecosistema y aquellas que cumplen al interior del bioterio.

5. ¿Qué ocurriría en el sistema si el funcionamiento de algún elemento del bioterio se altera?

6. ¿Qué pasa si los niveles de agua y/o alimento al interior del bioterio no son los adecuados?

7. Dentro del macroproyecto propuesto para la conservación de los ecosistemas paramunos ¿Cuál sería el aporte del bioterio a este objetivo?

\section{Parte 3. Comportamiento de las estructuras y sus interdependencias} funcionales.

1. Desde el punto de vista ecosistémico ¿explique los procesos mediante los cuales interactúan los factores bióticos y abióticos en los procesos de transformación de la energía? ¿Cómo interactúan los factores bióticos al interior del bioterio?

2. El ejemplo más común de reacciones endotérmicas y exotérmicas son expresadas químicamente a través de la siguiente ecuación $6 \mathrm{H} 20+6 \mathrm{CO} 2+$ $602+$ C6 H12 06 ¿cómo están involucrados la mariposa y las plantas en estos procesos?

3. Dentro de las diferentes formas de interacción ¿existen canales de retroalimentación en el bioterio?

4. Describa el proceso mediante el cual los ecosistemas paramunos contribuyen a lo formación de recursos hídricos Describa el papel que juegan los ecosistemas altoandinos en la regulación del ciclo hídrico

5. ¿Cómo interactúan con el ambiente natural los factores que tuvo en cuenta para la construcción del bioterio? 


\section{Apéndice 2}

Análisis objetivo.

\section{Resumen Análisis de Tarea: SRP "El Mariposario"} (ecosistemas).

Objetivo: evaluar la comprensión del funcionamiento de sistemas complejos

Dirigida a: estudiantes de los programas de Licenciatura en Ciencias Naturales y Educación Ambiental, y Biología.

\section{Fases de aplicación}

1. Registro de los datos sociodemográficos: consentimiento informado, nombre, apellido, género (masculino o femenino) fecha de nacimiento (Día/Mes/Año), institución educativa y curso.

3. Instrucciones verbales, se verifica que el participante ha comprendido la tarea y lo que debe hacer. El participante indica el momento de avanzar o retroceder para verificar, analizar o recordar información dada en la consigna introductoria.

5. Situación de resolución de problemas: de manera individual y escrita, el participante resuelve el problema presentada en un ambiente libre de ruido.

\section{Análisis subjetivo.}

6. Conclusión y cierre: se da por finalizada la tarea y se agradece al participante.

Exigencias cognitivas: Protegiendo las mariposas es una tarea que pretende evaluar la comprensión del funcionamiento de sistemas complejos a través del Modelo Estructura, Comportamiento y Función (ECF) planteado por Hmelo \& Silver (2004). Con la tarea, se rastrea el razonamiento deductivo del estudiante y la construcción conceptual basada en teorías de la biología y los sistemas complejos. Para resolver la tarea, el estudiante debe usar los siguientes procesos psicológicos: a) atención sostenida o vigilancia y mantenimiento de la atención durante un periodo de tiempo determinado; $b$ ) fluidez verbal para la correcta y rápida elocución del lenguaje oral; c) memoria para el almacenamiento y recuperación de información que juega un papel básico en los procesos de aprendizaje y pensamiento; d) planificación para desarrollar planes o prever los pasos para alcanzar un objetivo; e) razonamiento abstracto para resolver problemas lógicos, de prever y planear, es un pensamiento que funciona imaginando, o resolviendo problemas mentalmente; y f) razonamiento causal para identificar el origen de un suceso, sus posibles causas, fundamentos, relaciones e importancia de cada una de ellas.

\section{Tarea SRP "El Mariposario"}

Debido a diferentes actividades antrópicas en las zonas circundantes al parque se ha reducido la extensión de la flora aledaña. Las principales plantas afectadas han sido la chusqueatessellota (planta huésped que proporciona recurso para alimentar a la larva de la Lymanopodamirabilis)y los frailejones (fuente de alimento del adulto de la Lymanopodamirabilis). Se ha decidido construir el bioterio en condiciones in situ en un ecosistema altoandino, particularmente en la zona aledaña al Parque Natural Nacional Chingaza, el cual cuenta con 70 metros cuadrados, que usted podrá usar libremente, ya que es hábitat de la Lymanopodamirabilis.

A continuación, se detallan las plantas por metro cuadrado y el perfil de vegetación en la zona en la que se encuentran el bioterio: 1 frailejon (Espeleitamiradorensis); 2 Gaultheriaanastomosans(Familia Ericaceae); 1 romero de páramo (Diplostephiumrosmarinifolium); 2 arbustos de agraz (Vacciniumfloribundum); 3 arbustos 
de Gaultheriamyrtilloides(Familia Ericaceae); 4 Geraniummulticeps(Familia Geraniaceae); 6 helechos (Blechnum cf. Loxense); 20 Paepalanthus dendroides (Familia Eriocaulaceae); 1 Chusqueatessellata; 9 Senecioformosoides(Familia Asteraceae); 5 arbustos de Ribesandicola/Familia Grossulariaceae); 5 arbustos de Rubusacanthophyllos(Familia Rosaceae); 15 helechos de Lycopodiumclavatum; 4 Peperomiamicrophylla(Familia Piperaceae); 3 Carexjamenosi(Familia Cyperaceae).

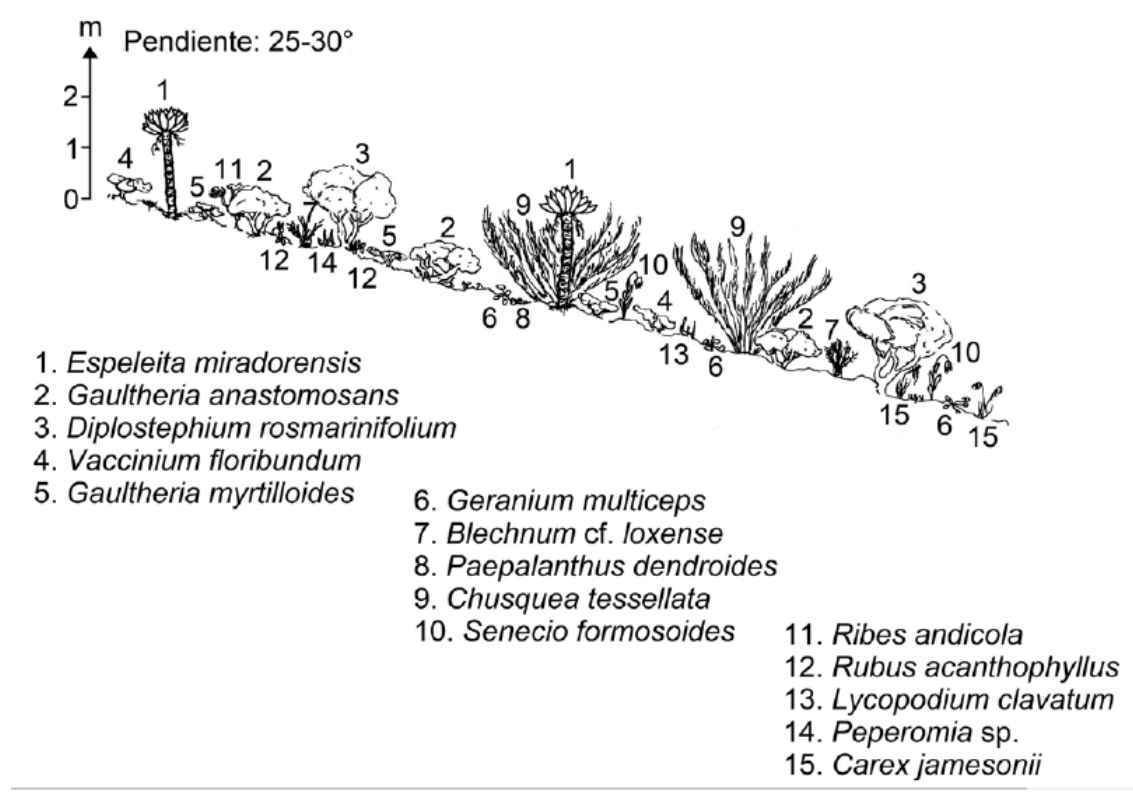

Figura 3. Perfil de la vegetación del área aledaña al bioteri. Tomado de Ávila \& Triviño (2019).

Los investigadores han utilizado al interior del bioterio la proporción de plantas por metro cuadro que se encuentran en el hábitat de la Lymanopodamirabilis. Durante las primeras semanas de funcionamiento del bioterio se han observado grandes dificultades en su interior. Por un lado, se ha producido un desabastecimiento de fuentes de alimentos para las larvas y los adultos (imagos) de la Lymanopodamirabilis, lo que ha ocasionado pérdidas elevadas en el número de larvas. A esta situación, se suma que los adultos (imagos) han dejado de reproducirse, condición que representa una grave amenaza para el bioterio y el proyecto de conservación debido a su corto ciclo de vida. Además, las fuentes hídricas que alimentan el sistema de riego del bioterio son escasas y los investigadores han descubierto que estas fuentes también son compartidas por las actividades antrópicas previamente mencionadas.

Como parte del equipo de investigación, su misión consiste en:

a) Formular una hipótesis sobre lo que está ocurriendo al interior del bioterio.

b) Proponer un experimento que compruebe su hipótesis.

c) Generar un plan en el que programe las acciones necesarias para resolver la compleja situación del bioterio.

En el desarrollo de la actividad puede incluir diagramas, figuras, dibujos o cualquier elemento que considere necesario para que su solución sea lo más detallada. 